\title{
An Unusual Cause of Haemorrhagic Cystitis in a Teenager with Medulloblastoma
}

\author{
Daniel Slack ${ }^{1}$, Satyajit Ray ${ }^{1}$, Boma Lee ${ }^{1}$, and Jairam Sastry ${ }^{1}$ \\ ${ }^{1}$ Royal Hospital for Children
}

May 19, 2020

\begin{abstract}
Haemorrhagic cystitis is a life-threatening condition in which the transitional epithelium and blood vessels of the bladder necrose leading to severe haematuria, abdominal pain and voiding lower urinary tract symptoms. Aetiology includes chemotherapy (cyclophosphamide, ifosfamide, busulfan), radiotherapy or infectious agents. We present a paediatric case of a 15-year-old boy with medulloblastoma who developed haemorrhagic cystitis following cisplatin chemotherapy. All other causes were ruled out and it is therefore likely that the agent in this case was cisplatin, which has never had haemorrhagic cystitis reported as a side effect. We also suggest a mechanism for urothelial injury centred around OCT-2 receptors.
\end{abstract}

\section{An Unusual Cause of Haemorrhagic Cystitis in a Teenager with Medulloblastoma}

Haemorrhagic cystitis (HC) is a life-threatening condition in which the transitional epithelium and blood vessels of the bladder necrose leading to severe haematuria, abdominal pain and voiding lower urinary tract symptoms ${ }^{1}$. Often occurring in immunosuppressed patients, aetiology includes chemotherapy (cyclophosphamide, ifosfamide, busulfan), radiotherapy or infectious agents (bacterial urinary tract infection, adenovirus, BK polyomavirus (BKV) and cytomegalovirus) ${ }^{2}$. In many cases no obvious cause can be identified, but a number of risk factors have been reported in the literature that predispose these patients to developing $\mathrm{HC}$. The incidence has a wide range between $3 \%$ and $35 \%$, dependent on aetiology and disparity between HC definitions used in research ${ }^{3}$. Mild $\mathrm{HC}$ often resolves spontaneously without complications, but moderate to severe $\mathrm{HC}$ may result in life-threatening complications or death.

The mainstay of treatment lies with blood-clot evacuation with wide bore urinary catheters and continuous bladder irrigation, hyperhydration with intravenous fluids and supportive red cell and/or platelet transfusion $^{3}$. Oxybutynin often provides benefit and sodium pentosan polysulphate (SPP) can be used to replace the damaged glycosaminoglycan layer in the bladder ${ }^{4,5}$. Otherwise management is specific to the underlying cause. For example, Mesna is given alongside oxazaphosphorine chemotherapy as hepatic metabolism of these agents forms acrolein, which is excreted in urine and leads to ulceration and necrosis of the urothelium. Mesna binds acrolein and prevents this. Viral infections can be managed supportively or treated with antivirals. Evidence is emerging which supports the use of hyperbaric oxygen in radiationinduced $\mathrm{HC}^{3}$. Treatments such as oestrogen, intravesical alum, or formalin are sometimes considered in difficult $\operatorname{cases}^{6,7,8}$.

We present a paediatric case of $\mathrm{HC}$ secondary to either cisplatin or lomustine, both of which have never been reported to have $\mathrm{HC}$ as a side effect.

\section{Case}

A 15-year-old boy presented with severe headache and vomiting. Emergency CT head showed a mass in the patient's fourth ventricle in the posterior fossa, later identified as a Classic Medulloblastoma (WHO 
grade IV, WNT-activated, CTNNB1 mutated, M1 Chang stage disease) by biopsy. The patient underwent a right frontal EVD insertion, posterior fossa craniotomy and resection and a haematoma evacuation. He was eligible for, and underwent, proton beam therapy to the craniospinal axis with boost to the posterior fossa. Following this, an MRI showed no recurrent or residual disease and he proceeded with post-operative chemotherapy.

Following the CCLG standard risk guidelines, he was treated with lomustine, vincristine, cisplatin and cyclosphosphamide. Details of the chemotherapy are shown in table 1.

In cycle 1, vincristine, lomustine and cisplatin were given on day 1 . At day 2, the patient developed severe haematuria with gross clots, causing significant dysuria. The patient declined catheterisation and was managed with intravenous fluids at $150 \%$ maintenance and analgesia.

Creatinine increased from $56 \mathrm{umol} / \mathrm{L}$ pre-chemotherapy to $73 \mathrm{umol} / \mathrm{L}$. He had a prolonged prothrombin time (15s) and was given $5 \mathrm{mg}$ of vitamin $\mathrm{K}$ twice daily. Platelet count was normal.

He subsequently developed significant hesitancy, urgency and dysuria in addition to passing more clots. The patient was passing urine every 15 minutes at its worst, associated with significant pain. A clinical diagnosis of $\mathrm{HC}$ was made. He required red-cell transfusion for a haemoglobin of $60 \mathrm{~g} / \mathrm{L}$ and was treated with oxybutynin, SPP and morphine patient-controlled analgesia (PCA) on the advice of urology. A repeat ultrasound at day 9 showed a thickened, hyperaemic bladder wall with echogenic debris, in keeping with HC. Catheterisation was felt not to be in the patient's best interest as despite pain he was passing urine regularly.

Urine culture was negative with no presence of pyuria or organisms on microscopy. Urine dips were positive for blood and white cells, but no nitrites. Urine (x2) and plasma samples for BKV were negative. Cytomegalovirus (CMV), Epstein-Barr virus (EBV) and adenovirus serology were negative. Throughout this episode, the patient's neutrophils remained within normal range. C-reactive protein was $32 \mathrm{mg} / \mathrm{L}$ at its peak.

The patient recovered by day 14. Frank haematuria stopped at day 10. Oxybutynin, PCA and SPP were weaned and further vincristine was delayed to day 21 . The patient did not experience a recurrence of HC.

\section{Discussion}

Our patient developed grade 3 CTCAE HC, which resolved completely with supportive care. Chemotherapyinduced $\mathrm{HC}$ generally resolves upon withdrawal of the offending agent, along with the treatments described above. It has a favourable prognosis when compared to $\mathrm{HC}$ caused by pelvic radiotherapy, which can recur for many months post-treatment. Complications are rare in chemical-induced HC but can include acute kidney injury requiring dialysis, urinary tract obstruction/infection, bladder perforation and death ${ }^{4}$. Long-term effects include bladder and upper tract fibrosis as well as reduced bladder capacity. In paediatric patients, increased mortality is linked to catheterisation, need for dialysis and presence of $\mathrm{BKV}^{9}$.

Infective agents were ruled out in this case. Urine culture was negative with no evidence of pus or white cells/organisms on microscopy. The absence of pyuria has a negative predictive value of near $90 \%^{11}$. BKV, adenovirus and CMV have been implicated in the pathogenesis of $\mathrm{HC}^{12,13}$ and serology for all was negative.

Pelvic radiotherapy is a known cause of HC. Proton beam therapy, which is increasingly used for medulloblastoma in a paediatric population, allows for more precise treatment than traditional photon radiotherapy due to the Bragg peak phenomenon ${ }^{14}$ and therefore reduces the damage done to adjacent organs and tissues. Craniospinal irradiation is carried out to the level of L1 vertebrae and is therefore sufficiently removed from the bladder to rule out. There is no evidence in the literature that $\mathrm{HC}$ can be caused by distant radiotherapy.

A literature review regarding each of the medications the patient was taking (levomepromazine, omeprazole, ondansetron and paracetamol) revealed one unrelated result when searched with HC on Pubmed. Accidental administration of cyclophosphamide was excluded after thorough investigation by pharmacy and nursing staff. 
It is possible that the chemotherapy agents the patient received before $\mathrm{HC}$ developed are culprits. Vincristine is mostly hepatically metabolised ${ }^{15}$ and is commonly used in many chemotherapeutic regimes and is therefore unlikely to be the cause. Lomustine is metabolised renally, but does not typically cause any renal issues, unless used for a prolonged period ${ }^{16}$.

Cisplatin is a platinum-based alkylating agent used for many malignancies and is well documented to cause nephrotoxicity due to accumulation in renal tubules ${ }^{17}$. The co-transporter protein OCT-2 has been identified as a key protein for the uptake of cisplatin into renal cells, and cisplatin competitively inhibits other substrates that are normally taken up via OCT-2. Inside the cell, there are a myriad of mechanisms whereby cisplatin can cause apoptosis/necrosis of the cell, including upregulation of $\mathrm{p} 53^{18}$. OCT-2 receptors are also found in the urothelium ${ }^{19}$, so it follows that cisplatin could be taken up in a similar way in the bladder and cause inflammation and necrosis, leading to HC. In addition, carboplatin, a similar akylating agent, which can be substituted with cisplatin in the CCLG treatment guideline, has been mentioned in one paper as a potential cause $^{3}$. Another case reports HC following combination chemotherapy with bleomycin, vinblastine, cisplatin and etoposide ${ }^{20}$.

Given the number of causes for HC, it is possible that cisplatin-induced HC has been underreported. Adenovirus is common among the general population and it is not hard to imagine a rare patient with cisplatininduced $\mathrm{HC}$ being co-infected with adenovirus and the condition being falsely attributed to the known cause.

\section{Conflict of Interest}

The authors know of no conflict of interest in publication of this work.

\section{References}

1. Sahu KK, Prakash G et al. A Rare Case of Haemorrhagic Cystitis in Allogeneic Haematopoetic Stem Cell Transplant Patient. Indian J of Haematology and Blood Transfusion 2016; 390: 196-200.

2. Ebiloglu T, Kaya E, Yilmaz S, Özgür G, Kibar Y. Treatment of Resistant Cyclophosphamide Induced Haemorrhagic Cystitis: Review of Literature and Three Case Reports 2016; 10(4): 15-16.

3. Payne H, Adamson A et al. Chemical and Radiation Induced Haemorrhagic Cystitis: Current Treatments and Challenges. BJU International 2013; 112(7): 885-897.

4. Seber A, Shu XO, Defor T, Sencer S, Ramsay N. Risk Factors for Severe Haemorrhagic Cystitis Following BMT. Bone Marrow Transplantation. 1999. 23: 35-40.

5. Decker DB, Karam JA, Wilcox DT. Paediatric Haemorrhagic Cystitis. Journal of Paediatric Urology 2009; 5: 254-264.

6. Heath JA, Mishra S, Mitchell S, Waters KD, Tiedemann K. Estrogen as treatment of hemorrhagic cystitis in children and adolescents undergoing bone marrow transplantation. Bone Marrow Transplant 2006; 37: 523-26.

7. Miller J, Burfield GD, Moretti KL. Oral conjugated estrogen therapy for treatment of haemorrhagic cystitis . J Urol 1994; 151: 1348.17.

8. Mukamel E, Lupu A, deKernion JB. Alum irrigation for severe bladder hemorrhage. J Urol 1986; 135: 784 .

9. Au JK, Graziano C et al. Urologic Outcomes of Children with Haemorrhagic Cystitis After Bone Marrow Transplant at a Single Institution. Urology 2016; 101: 126-132.

10. Duthie G, Whyte L, Chandran H, Lawson S, Velangi M, McCarthy L.Introduction of Sodium Pentosan Polysulphate and Avoidance of Urethral Catheterisation: Improved Outcomes in Children With Haemorrhagic Cystitis Post Stem Cell Transplant/Chemotherapy. J Paediatric Surgery 2012; 47(2): 375-9.

11. Simerville JA, Maxted WC, Pahira JJ. Urinalysis: A Comprehensive Review. Am Fam Physician 2005; 71: 1153-1162.

12. $\mathrm{Hu} \mathrm{J}, \mathrm{Li} \mathrm{S}$ et al. Incidence, Risk Factors and the Effect of Polyoma Virus Infection in Haematopoetic Stem Cell Transplant Recipients. J International Medical Research 2017; 45(2): 762-770.

13. Lynch JP, Fishbein M, Ecchavaria M. Adenovirus. Seminars in Respiratory and Critical Care Medicine 2011; 32(4): 494-511. 
14. Liu H, Chang JY. Proton Therapy in Clinical Practice. Chinese Journal of Cancer 2011; 30(5): 315-326.

15. EMC. Vincristine Sulphate $1 \mathrm{mg} / \mathrm{ml}$ for Injection. Authorised 2/12/2008. Accessed 10/12/2019 athttps://www.medicines.org.uk/emc/product/1502/smpc.

16. EMC. Lomustine "Medac" 40mg. Authorised 25/08/2006. Accessed 10/12/2019 athttps://www.medicines.org.uk/emc/product/1401/smpc.

17. Crona DJ, Faso A et al. A Systematic Review of Strategies to Prevent Cisplatin-Induced Nephrotoxicity. Oncologist 2017; 22(5): 609-619.

18. Miller RP, Tadagavadhi RK, Ramesh G, Reeves WB. Mechanisms of Cisplatin Nephrotoxicity. Toxins 2010; 2(11): 2490-2518.

19. Leonhauser D, Kranz J et al. Expression of Components of the Urothelial Cholinergic System in Bladder and Cultivated Primary Urothelial Cells of the Pig. BMC Urology 2019; 19: 62.

20. Cantwell BM, Harris AL, Patrick D, Hall RR. Haemorrhagic Cystitis After I. V. Bleomycin, Vinblastine, Cisplatin and Etoposide for Testicular Cancer. Cancer Treatment Reports 1986; 70(4): 548-9.

\section{Hosted file}

Table 1 - AG Final.docx available at https://authorea.com/users/323850/articles/452314-anunusual-cause-of-haemorrhagic-cystitis-in-a-teenager-with-medulloblastoma 